\title{
Experimental investigation on the folding of axially crushed hexagonal tubes
}

\author{
M. R. Said ${ }^{1}$, A. A. Mokhtar ${ }^{1}$, A. Alias ${ }^{2}$ \& A. Ibrahim ${ }^{3}$ \\ ${ }^{I}$ Faculty of Mechanical Engineering, Kolej Universiti Teknikal, \\ Kebangsaan, Malaysia, \\ ${ }^{2}$ Faculty of Mechanical Engineering, Universiti Teknologi, Malaysia \\ ${ }^{3}$ Universiti Utara, Malaysia
}

\begin{abstract}
The objective of this paper is to investigate the folding mechanism of an empty hexagonal tube made of mild steel with respect to the energy absorption. This includes the determination of the plastic wavelength, type of deforming mode and the mean load of the tube subjected to an axial loading. The introduction of chamfer at one end of the tube is also examined and studied. The comparison load-displacement curves between experiment and Finite Element Analysis (FEA) are made. The experimental mean load is also compared with theoretical analysis. The response of the hexagonal tube subjected to axial loading is observed through experiments. The formation of plastic fold length is observed and measured. The mean load and area under the load-displacement curve are calculated to obtain the energy absorption. By introducing the chamfer at one end, the deforming pattern can be determined, i.e. in-out chamfer gives a diamond mode while all-out chamfer gives a concentina mode. The FEA loaddisplacement curve agrees well with the experiment. However, the predicted mean load is overestimated, while the equivalent circular cylinder underestimates the experimental result.
\end{abstract}

Keywords: energy absorption, hexagonal tubes, triggering, and crushed.

\section{Introduction}

Energy absorption systems made of thin-walled circular tubes and tube nests have been given significant attention by researchers for the past few decades. In particular, the initiation progressive axisymmetric and non-axisymmetrical 
folding in tubes has been a subject of intense investigation by many investigators. Alexander [1] was first to present an analysis for the prediction of a mean crushing load, for a tube subjected to axial compression. He managed to obtain an expression for the folding length and the mean crushing load by implementing global energy balance. However, he assumed the tube to be folding into axisymmetric rings, in which the tube wall moves out of its initial position. But in reality there is outward as well as inward movement. This anomaly was first addressed by Reddy and Reid [2] albeit by an over prescribed hinge (four) mechanism. Wierzbicki et al [3] presented a more realistic three hinges mechanism in the form of a concentina collapse mode. The mechanism allows for radially inward as well outward folding in a proportion relative to the tube generator. The outer portion of the folding length relative to the total (inward plus outward) length was defined by the eccentricity factor. The mean load deduced was independent of the eccentricity factor, which was indeterminate. Singace et al [4] further modified Wierzbicki's analysis enabling the determination of the eccentricity factor, which was in good agreement with the experimental results. The absolute mean crushing load produced by Singace et al [4] was similar to that of Wierzbicki et al [3] except for an added factor 5.632. These and other analysis are applicable only on the second and subsequent folds. The formation of the first fold is unique and there appears to be no analysis yet to describe it.

Pugsley and Macaulay [5] observed diamond type folding in thinner tubes and derived a semi-empirical expression for the mean load. Horton et al [6] reported the findings of an extensive experimental study of quasi-static axial buckling of thin-walled cylindrical tubes, concluding that the buckling mode changed from axisymmetric to non-symmetric patterns if the geometric parameter, radius over wall thickness, $t$ increases. Johnson et al [7] developed, a mechanism of inextensional mode of deformation of thin-walled tube under axial loading which involved the stationary and travelling hinges in the diamond mode and derived an expression for the mean crushing load. However, they [7] did not correlate the change of concentina to diamond mode. Tvergaard [8] investigated the influence of buckling pattern localisation, which could cause the transition of diamond mode to axisymmetric mode. The dependence of folding mode on tube geometry has been investigated experimentally by Andrews et al [9].

Square, rectangular, hexagonal are more popular than circular tubes in automobile industry. Early investigations $[10,11]$ were concerned with rectangular tubes made of sheet metal and were aimed to understand the behaviour of vehicle body shells. Wierzbicki and Abramowicz [12] formulated kinematically admissible global deformation mechanisms for thin-walled rectangular tubes comprised of flat plates. Reid and Reddy [13], Reid et al. [14] and Reddy and Al-Hassani [15] have shown that introducing different fillers like foam or wood increase the overall energy absorption of the tubes. Said [16] presented experimental measurement of elastic half wavelength and plastic fold length during the crushing of rectangular tubes. No work has been found to study the effect of trigger by introducing the chamfer in one end tube. Abramowicz and Wierzbicki [17] developed a mechanism for predicting the crush behaviour of 
multicorner columns with an arbitrary corner angle. This was applied to find crushing strength of hexagonal tube under axial compression and produce a simple expression for the mean crushing load.

As the hexagonal consists of 6 sides and circular is infinity sides, the correlation may be made by assuming the hexagonal sides as an equivalent circular cylinder. The objective of this paper is to investigate the folding mechanism of empty hexagonal tube made of mild steel with respect to the energy absorption. The effect of triggering with mean load and plastic folding is also examined.

\section{Experiment}

Axial compression tests were carried out on as-received $170 \mathrm{~mm}$ and $200 \mathrm{~mm}$ long hexagonal tubes made from the same tube. Longer specimens were labelled as HEX1 to HEX12 and shorter (170 mm long) specimens as HEX170. Some of them were marked with $4 \mathrm{~mm}$ grid circles and some chamfered at one end in order to trigger the mode of deformation. Distance of $4 \mathrm{~mm}$ is measured from a point of arc intersection to the next point. All the axial compression tests were on specimens in the as-received state only. Tests were carried out on a $200 \mathrm{kN}$ universal testing machine at a loading rate of $10 \mathrm{~mm} / \mathrm{min}$.

\section{Observations and discussions}

This section describes the influence of triggering, the characteristic of loaddisplacement curves, and the effect of chamfer. This includes the determination of the plastic fold length, mean load and energy absorbed and also compared with the circular tube under axial loading.

\subsection{Triggering the mode of deformation}

Mode of deformation of hexagonal tube under axial loading can be triggered by introducing the chamfer at one end. Without chamfering, the tube deforms in uncertainty manner, i.e. may deform in concentina or diamond or mixed mode. Experiments have shown that by introducing the chamfer, the mode of collapse can be determined. However, the edge of the tubes under investigations, have to be suitably chamfered. The chamfer was made to half of the original thickness of the tube and with an angle of approximately $45^{\circ}$ as shown in Figure 1 . This results in the axial end forces applied being offset with respect to the centreline of the tube wall. The larger the chamfer is the larger the eccentricity. Experiments have shown that if the chamfering one side only of all end faces (either inner or outer ends), tube collapses into concentina mode.

With external chamfers on all faces (referred to as all-out chamfers), the first buckle was outwards and vice versa. However, if adjacent faces were chamfered on opposites edges (i.e. one on the outside and the other on the inside, referred to as in-out chamfers), the tubes were seen to fold into the diamond mode. In the inout chamfered tubes, fractures were observed along the corners when the chamfers were extended over the whole face width. By chamfering only over the central region, such corner failures were prevented. 


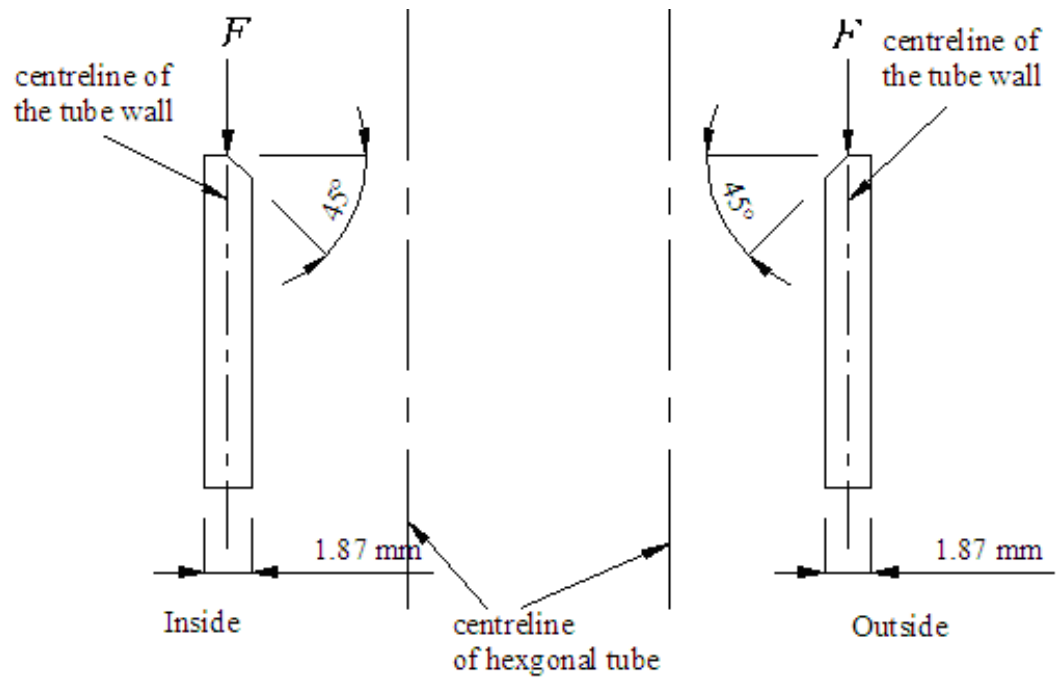

Figure 1: A schematic view of the tube wall, showing the chamfered angle.

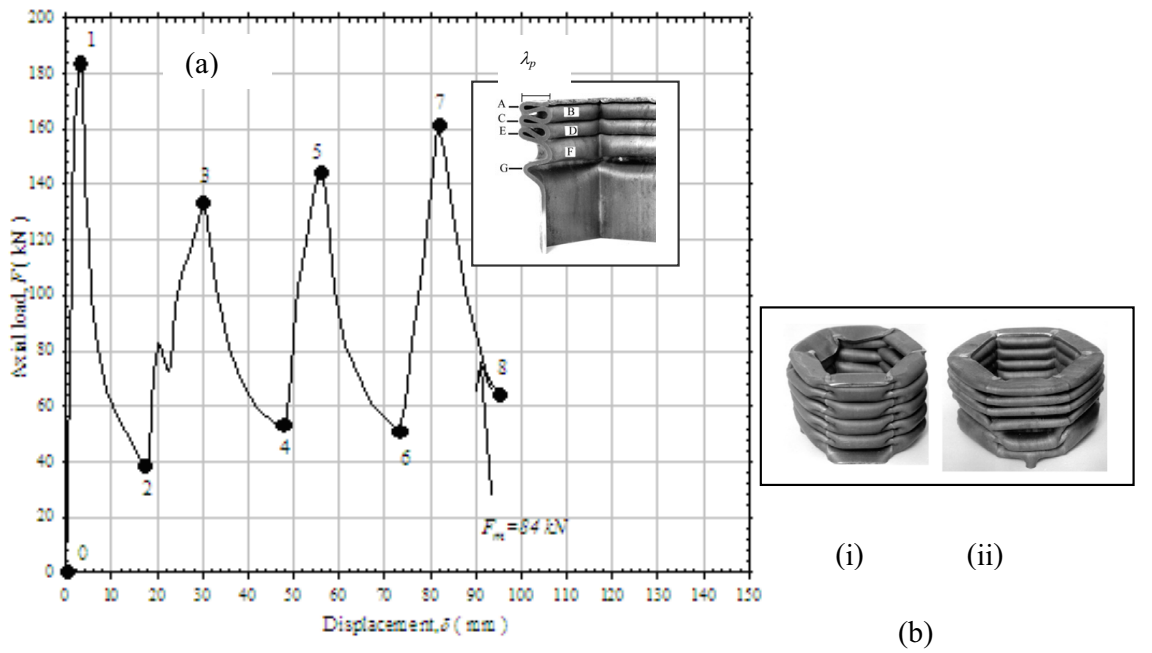

Figure 2: (a) A typical load-displacement curve for a single hexagonal tube (HEX170). Insert: A one-sixth segment of a deformed specimen. (b) Deformed unchamfered tubes (i) Diamond mode (ii) Concentina mode.

\subsection{The load-displacement characteristic for unchamfered specimens}

Figure 2 shows a typical load-displacement trace for an unchamfered specimen marked as HEX170 and the deformed tubes. A cross-section of the crushed specimen is shown in the inset. During compression, just before the peak load (point 1 in Figure 2a), a series of elastic ripples were noticeable in the sides of 
the hexagonal tube. It is noticed that, the peak load is $184 \mathrm{kN}$. Ripples disappeared when the load dropped (point 1 to 2 ) and were not noticeable afterwards. They reflect the state of buckling and influenced mostly by strainhardening characteristics of these tubes. At the peak load the tube buckled locally at the top end and initiated a plastic fold. The faces were observed to buckle before the corners collapsed indicating the start of plastic fold. The first fold was followed by a series of load fluctuations. Each cycle in the fluctuations (e.g. 3-5, 5-7 etc) corresponds to a plastic fold that formed in the tube at section labelled as A, B, C, D, E, F and G shown in the inset. The number of peaks in the load-displacement curve indicates the number of folds formed. The mean load during compression was calculated as the ratio of the area under the curve and the displacement is almost the same for all folds. A secondary (minor) load fluctuation was observed between the first and second primary peak only. Such fluctuation was not seen beyond the second peak. A characteristic (plasticbuckling) wavelength can be identified as, $\lambda_{p}(=2 H)$ as shown in the inset in which mode of deformation seen is of a concentina type, in which all the sides of the tube buckle in phase i.e. inward or outwards simultaneously. The plastic wavelength can be estimated from experiment, as the average length per fold in the deformed specimens. It was found that, $\lambda_{p}=33 \mathrm{~mm}$, this also being approximately the displacement difference between two adjacent peaks in the load-displacement characteristic (Figure 2a). A detailed examination of the deformed specimen also indicated that, the first fold was at about $16 \mathrm{~mm}$ from top end as shown in the inset. From the calculation, it is noticed that the mean load is about $84 \mathrm{kN}$. Some specimens exhibited progressive incomplete diamond and concentina type folding patterns in a fold. The diamond type is where in adjacent sides of the tube were buckling out of phase (i.e. one inward and the other outward etc). Incomplete mode is a mixture of half concentina and half diamond in one plastic fold. For example, specimen HEX1 deformed in four incomplete diamond and concentina folds as can be seen from photograph in Figure 2b(i-ii).

In some specimens, concentina fold formation was seen in the initial few folds and then followed by incomplete diamond and concentina folding mechanism was seen. For example in specimen HEX7, Figure 2b(ii), three concentina folds are followed by two folds of incomplete diamond and concentina. None of the specimen was seen to fold in mixed mode. Mixed mode is a complete concentina and diamond folds in the same tube. This concludes that unchamfered specimens exhibit uncertainty of folding mechanisms. A summary of results that includes mean load and plastic fold length for the entire specimen tested is shown in Table 1. This includes the specimens in which triggers are introduced to induce specific mode, i.e. concentina or diamond type of deformation.

\subsection{The load-displacement characteristic for chamfered specimens}

Figure 3a-b shows typical load-displacement curve for in-out and all-out chamfered hexagonal tubes subjected to axial loading. In-out chamfered 
specimen exhibits diamond mode, while all-out in concentina mode. The sequence of deformation for the case of all-out chamfers (point 0 to 15) is shown in Figure 4 corresponding to Figure $3 \mathrm{~b}$. Point 1 of the same figure indicates the peak load and the initiation of first plastic fold. The peak load shows $165 \mathrm{kN}$ for both in-out and all-out chamfers.

Table 1: $\quad$ Summary of results of hexagonal tubes subjected to axial crushing.

\begin{tabular}{|c|c|c|c|c|}
\hline Spec. no. & $\begin{array}{c}\text { average } \\
\lambda_{p} \text { at mid } \\
\text { face } \\
(\mathrm{mm})\end{array}$ & $\begin{array}{c}\text { mean } \\
\begin{array}{c}F_{m} \\
(\mathrm{kN})\end{array}\end{array}$ & $\begin{array}{c}\text { Energy } \\
\text { absorbed } \\
W(\mathrm{Nm}) \\
\text { at } \delta=100 \\
\mathrm{~mm}\end{array}$ & $\begin{array}{c}\text { Mode } \\
\text { of } \\
\text { deformation } \\
\text { and length of specimen, } L\end{array}$ \\
\hline HEX170 & 33 & 84 & 8401 & $\begin{array}{c}\text { Concentina, } L=170 \mathrm{~mm} \\
\text { (unchamfer) }\end{array}$ \\
\hline HEX1 & 36 & 84 & 8376 & $\begin{array}{c}4 \text { folds incomplete } L=200 \\
\text { mm (unchamfer) }\end{array}$ \\
\hline HEX2 & 35 & 84 & 8372 & $\begin{array}{c}4 \text { folds incomplete } L=200 \\
\text { mm (unchamfer) }\end{array}$ \\
\hline HEX3, 8, & 36 & 85 & 8488 & $\begin{array}{c}\text { Concentina, } L=200 \mathrm{~mm} \\
\text { (All-out chamfer) }\end{array}$ \\
\hline HEX4 & - & - & - & $\begin{array}{c}\text { Broke halfway, } L=200 \mathrm{~mm} \\
\text { (in-out chamfer) }\end{array}$ \\
\hline HEX5 & 36 & 86 & 8592 & $\begin{array}{c}\text { Concentina, } L=200 \mathrm{~mm} \\
\text { (all-out chamfer) }\end{array}$ \\
\hline HEX6 & - & - & - & $\begin{array}{c}\text { Broke halfway, } L=200 \mathrm{~mm} \\
\text { (in-out chamfer) }\end{array}$ \\
\hline HEX7 & 34 & 85 & 8477 & $\begin{array}{c}3 \text { folds Concentina, } 2 \text { folds } \\
\text { incomplete, } L=200 \mathrm{~mm}\end{array}$ \\
\hline HEX10 & 44 & 80 & 7939 & $\begin{array}{c}\text { Diamond, } L=200 \mathrm{~mm} \\
\text { (in-out chamfer) }\end{array}$ \\
\hline
\end{tabular}

This means that insignificant effect on peak load for in-out and all-out chamfers, as the initial compression areas are the same. However, the load fluctuation (amplitude) for in-out chamfer is smaller than all-out chamfer by about $25 \mathrm{kN}$. Referring to Figure 3b, two secondary peaks (point 3 and 5) exist in load-displacement curve between the first and second primary peak and one between second and third primary peak. But, the secondary peaks were not seen after the third primary peak (point 8). The existing of two secondary peaks may be due to the plastic fold in contact with the top platen. However, the secondary peaks were not seen for the case of in-out chamfered specimen. A summary of results that includes the mean load, and plastic wavelength for all the specimens also is included in Table 1. It shows no significant change on with respect to mean load and plastic folding length for concentina mode for the case of all-out chamfered. 


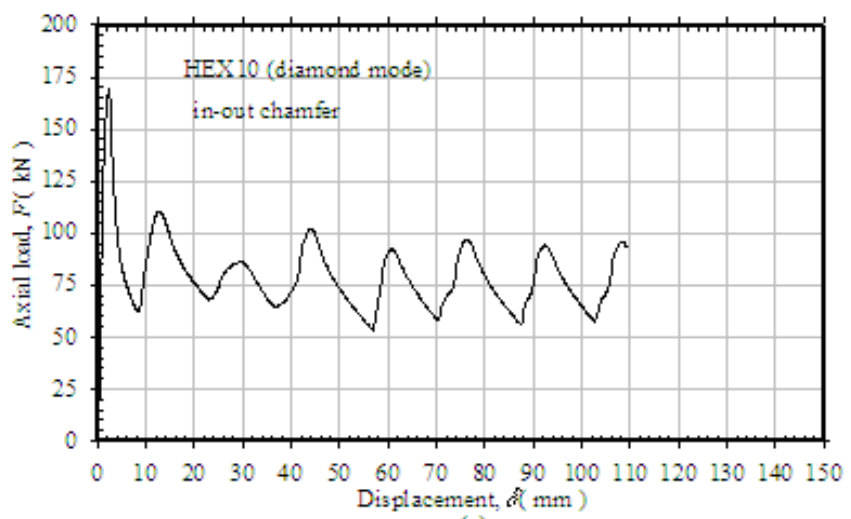

(a)

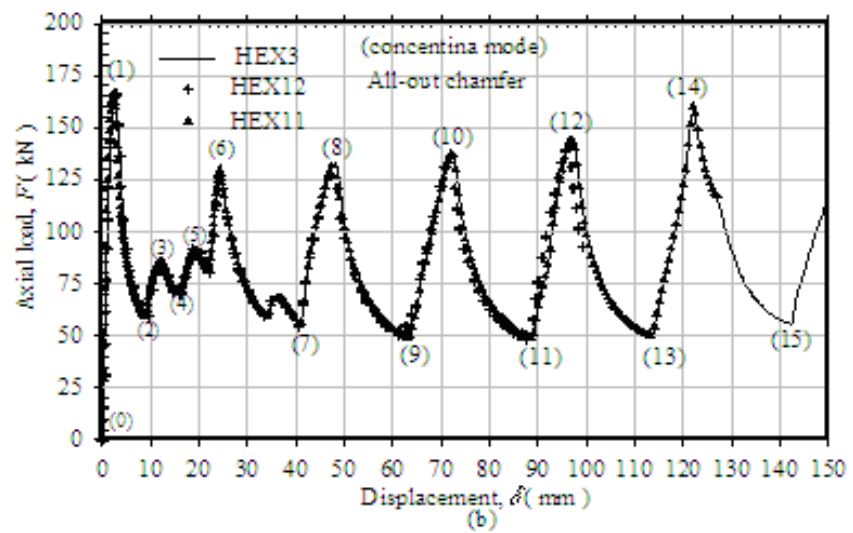

Figure 3: Load-displacement curve of hexagonal chamfered tubes under quasi-static compression (a) In-out chamfer (b) All-out chamfer.

However, in-out chamfer produces diamond mode, which gives the lower mean load, thus gives less energy absorption. Figures 5a-b show the typical cross-sectional views of the deformation patterns of all-out and in-out chamfered specimen crushed under nominally identical conditions. Figure 5a illustrates an all-out chamfered specimen, exhibiting concentina folds, while Figure 5b shows cross-section view of the diamond mode seen in the crushed specimen with inout chamfers.

\subsection{Effect of chamfer}

The effect of chamfer is observed for both all-out and in-out chamfer. The significant effect of all-out chamfer is noticed in the load-displacement curve (Figure $3 b$ ), which shows the existing of two secondary peaks at point 3 and 5 . Figure $3 \mathrm{~b}$ also show the repeatability of the peaks. No secondary peak has been found in in-out chamfer. The reason of non-existing of the secondary peak has not been explored. However, they also appear in unchamfered tubes but only one secondary peak exists at random. In general, from energy absorption point of view, the all-out chamfered tubes give a higher value of energy absorption. 


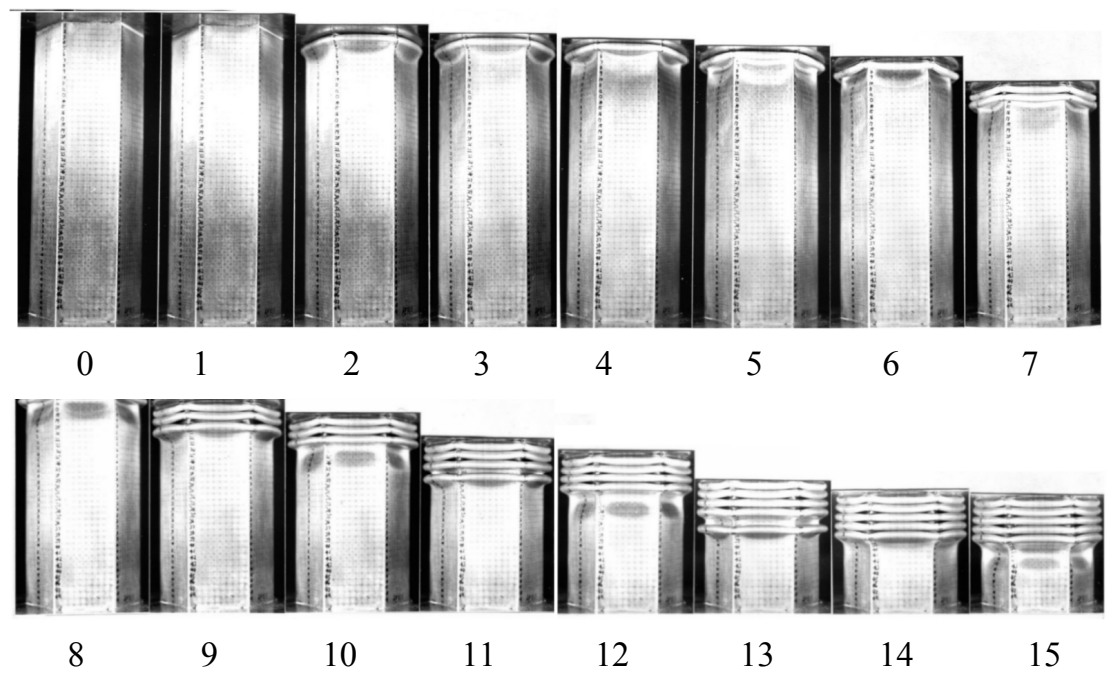

Figure 4: Sequence of deformation of axially crushed tube with all-out chamfer.
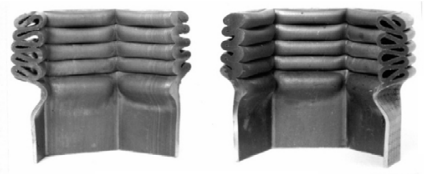

(a)
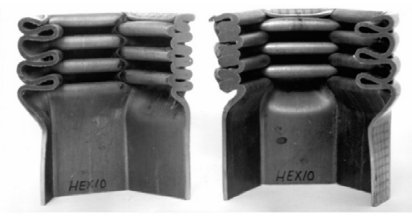

(b)

Figure 5: Typical cross-sectional views showing deformation pattern.

\subsection{Comparison with FEA and equivalent circular cylinder}

Figure 6 is the comparison of experimental load-displacement curves and those predicted by FEA [18,19], which are seen to be in good agreement. The FEA mean load is almost the same value with experiment. However the plastic fold length, $\lambda_{p}(=36 \mathrm{~mm})$ derived from the FEA $[18,19]$ overestimates the experiment by $10 \%$. The prediction of mean loads by various upperbound solutions for equivalent circular cylinder, Alexander [1] and Singace et al [4] and for hexagonal tubes, Abramowics and Wierzbicki [17] are also shown in the figure 6 . The radius of an equivalent circular cylinder is assumed to be equal side length, $b$ of hexagonal tubes. The mean load for the equivalent circular cylinders underestimates the experiment by about $30 \%$. This is not surprising because 
these solutions underestimate the mean load for the crushing of cylinder by about the same margin. Their plastic fold length, $\lambda_{p}[4]$ is also underestimated by about $30 \%$. The mean load predicted by the analysis of Abramowics and Wierzbicki [17] is $105 \mathrm{kN}$ which overestimates the experiment by about $25 \%$.

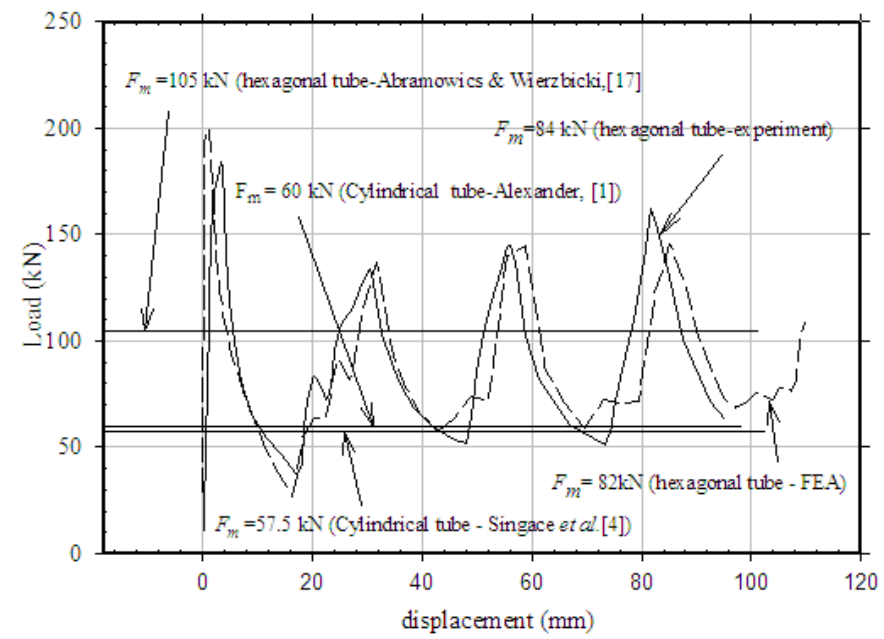

Figure 6: Load-displacement curves for axial compression quasi-static loading and comparison of the mean load, $F_{m}$ with previous researchers.

\section{Conclusion}

With chamfering, the tube deforms in certainty manner, i.e. in-out chamfer gives a diamond mode while all-out in concentina mode. However, a slightly higher energy absorption is obtained from concentina mode. It also shows no significant change on with respect to mean load and plastic folding length for concentina mode for the case of all-out chamfered. However, in-out chamfer produces diamond mode, which gives the lower mean load, thus gives less energy absorption. The mean load for equivalent circular cylinder underestimates the experimental result, however FEA give excellent results with experiment.

\section{References}

[1] Alexander, J. M. "An approximate analysis of the collapse of thin cylindrical shells under axial loading", Quart. J. Mech. Appl. Math, Vol.13, pp 10-14,1960

[2] Reddy, T. Y. and Reid, S.R., "Plastic folding of cylinders under axial compression" Presented at Euro Mech. $1^{\text {st }}$ European Solid Mech Conference at Munich, 1990 
[3] Wierzbicki, T., Bhat, S. U., Abramowicz, W. and Brodkin, D., “Alexander revisited-a two folding elements models of progressive crushing of tubes" Int. J. Solids Struct, Vol. 29, pp. 3269-3288, 1992

[4] Singace, A. A., El-Sobky, H. and Reddy, T. Y. "On the eccentricity factor in the progressive crushing of tubes" Int. J. Solids Struct Vol. 32, pp 3589-3602,1995

[5] Pugsley, A. and Macaulay, M. "The large scale crumpling of thin tubes", Quart J. Mech. Appl Maths, part 1, vol. 13, 1960

[6] Horton, W. H., Bailey, S. C. and Edwards, A. M. "Nonsymmteric buckle patterns in progressive plastic buckling", Experimental Mechanics, Vol. 6, No. 9. pp 433-444, 1966

[7] Johnson, W., Soden, P. D and Al-Hassani, S. T. S. "Inextensional collapse of thin-walled tube under axial compression", J. Strain Analysis Vol.12, No.4, 1977

[8] Tvergaard, V. "On the transition from a diamond mode to an axisymmetric mode of collapse in cylinder shells" Int. J. Solids Struct, Vol.19, No.10, pp 845-8561983

[9] Andrews, K. R. F, England G. L. and Ghani, E. "Classification of the axial collapse of cylindrical tubes under quasi-static loading" Int. J. Mech. Sci. Vol. 25, No. 9-10, pp. 687-696, 1983

[10] Macaulay, M. A. and Redwood, R. G. "Small scale model railway coaches under impact", Engineer, 1041-1046, 1964

[11] Postlethwaite, H. E. and Mills, B. "Use of collapsible structural elements as impact isolators with special reference to automotive application", $J$. Strain Analysis, 5, pp 58-73, 1970

[12] Wierzbicki, T. and Abramowicz, W. "On the crushing mechanics of thin walled structures”, J. Appl Mech, Vol. 50, pp 727-734, 1983

[13] Reid, S. R. and Reddy, T.Y. "Axial crushing of foam-filled tapered sheet metal tubes". Int. J. Mech. Sci., Vol.28, No.10, pp 643-656, 1986

[14] ] Reid, S. R., Reddy, T.Y. and Gray, M. D. "Static and dynamics axial crushing of foam-filled sheet metal tubes", Int. J. Mech. Sci., 28, pp. 295322, 1986

[15] Reddy, T. Y. and Al-Hassani, S. T. S., "Axial crushing of wood-filled square metal tubes”. Int. J. Mech. Sci., Vol.35, No.3/4, pp 231-246,1993

[16] Said, M. R. "Axial compression of empty and filled rectangular tubes". MSc. Dissertation, UMIST, U.K., 1988

[17] Abramowicz, W. and Wierzbicki, T. "Axial crushing of multicorners sheet metal columns” J. App. Mech., Trans ASME., 56, pp 113-120, 1989

[18] Said, M. R. "Energy absorption in certain cellular structures under uniaxial and biaxial loading" PhD. Thesis, UMIST, U.K., 2000

[19] Said, M. R. Ahmad R. and Alias A. "Finite Element Analysis of hexagonal tubes structure under axial loading" Proceeding in 1st International Conference On Safety and Security Engineering, pp 83-91, Rome, Italy, 2005 